\title{
Lipid profile comparison between temporal lobe epilepsy patients that are physically active and non-active.
}

\author{
Beatriz S. Braga*, Fernando Cendes.
}

\begin{abstract}
Epilepsy is the most frequent potentially severe chronic neurological disease. Temporal lobe epilepsy (TLE) is the most common form of focal epilepsy among adults. Faced with this, the main goal of this study is to verify if there is more occurence of MS (Metabolic Syndrome) on epilepsy patients and the relation between their levels of physical activity. In order to accomplish this, the comparison between lipidic levels from physically active and non-active TLE-patients was used, through blood collection and its analysis and through the appliance of IPAQ (International Physical Activity Questionnaire) and the PA levels classification. Our preliminary findings show that the epilepsy patients, even physically active, still present high levels of total cholesterol.
\end{abstract}

\section{Key words: \\ Epilepsy, Comorbidity, Physical Activity.}

\section{Introduction}

Temporal lobe epilepsy (TLE) is the most common form of focal epilepsy among adults and has high refractoriness to pharmacotherapy. The occurrence of comorbidity related to Metabolic Syndrome (MS) is higher among this population compared to the general population and can be related to the collateral effects of antiepileptic drugs (AED) and sedentarism. Although many studies prove the benefits of regular physical activity (PA) at preventing and treating $M S$, the clinical indication of PA is still not common for epilepsy patients. Therefore, this research goal is to analyze if there is difference in the frequency of MS between physically active and non-active epilepsy patients.

\section{Results and Discussion}

Volunteer patients with and without epilepsy were recruited from $\mathrm{HC}$ (Clinical Hospital of Unicamp), all individual were 40 - 60 years old, without (other) severe diseases and the group with epilepsy were receiving AED treatment. The data of the thirty-seven individuals were analyzed (27 patients with epilepsy and 10 healthy controls) through the International Physical Activity Questionnaire (IPAQ) and laboratorial tests. The data was analyzed and organized, from the questionnaire classification, and the individuals were divided in two groups, Epilepsy Patients (Epi) and Controls (C) which were afterwards again divided into two sub-groups, physically active epilepsy patients (EpiA) and sedentary epilepsy patients (EpiS), physically active controls (CA) and sedentary controls (CS).

We used the software Prism version 7 to verify if there was any significance relationship between the data. Through the data analysis, it can be noted that there is difference between patients with and without epilepsy.

In Figure 1 we can see that the total cholesterol is higher among the EpiA group than in the CA groups, and among EpiS group than in the CS group, however, there is no difference between the EpiA and EpiS groups. That implies that epilepsy patients have higher total cholesterol levels; therefore, suggesting that there is a higher frequency of MS in patients with epilepsy.

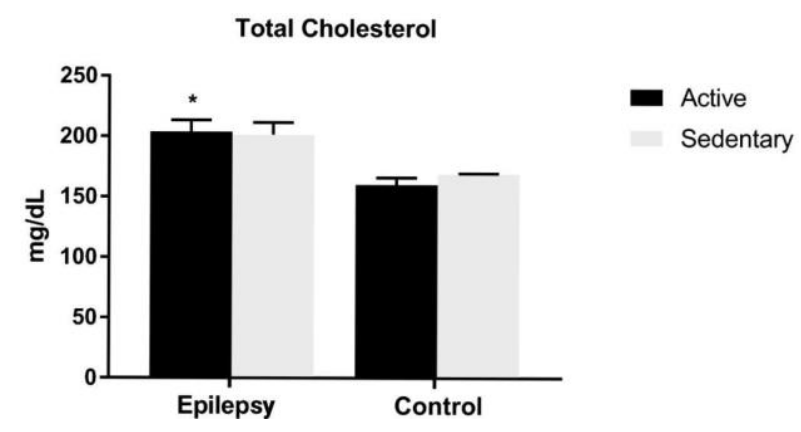

Figure 1: Epilepsy-Active, $\quad M=203.9, \quad S E M=9.564, \quad N=15$; Epilepsy-Sedentary, $\mathrm{M}=201.3, \quad \mathrm{SEM}=10.29, \mathrm{~N}=12$; ControlActive, $M=160, S E M=5.872, N=7$; Control Sedentary, $M=169$, $\mathrm{SEM}=0.577, \mathrm{~N}=3 .{ }^{*} \mathrm{P}=0.027$ related to EpiA e $\mathrm{CA}$.

$\mathrm{P}<0.05$. $\mathrm{M}=$ mean, $\mathrm{SEM}=$ standard error of mean

Despite the EpiA group being active, the cholesterol level was similar to the EpiS group. It is likely that there is a relationship between AEDs and cholesterol levels, which has to be analyzed further. The results presented until now reflect the research current stage, which will be expanded to include other variables, such as: glucose levels, HDL and LDL cholesterol and the relationship between calcium serum levels and the cardiorespiratory capacity from the maximum VO2 test.

\section{Conclusions}

Active epilepsy patients still present high levels of total cholesterol. Consequently, it is suggested that they should increase their PA levels, even if they are already active, to reduce the risks of MS case and improve their health as a whole.

\section{Acknowledgement}

To CNPq (National Board of Scientific and Technological Development) for the financial aid and to the volunteers that enabled the development of this research

Reynolds, E. H. Introduction: epilepsy in the world. Epilepsia, New York, v.43, Suppl 6, p.1-3,2002 